\section{NICE guidance delays mouth cancer diagnosis}

Oral facial surgeons say new NICE guidance for oral cancer ${ }^{1}$ is causing delays in diagnosis and treatment. Writing up their findings in the British Journal of Oral and Maxillofacial Surgery (BJOMS), they say that the new referral guidance does not have a defined referral pathway between doctors and dentists for some patients ${ }^{\star}$ with suspected cancer. ${ }^{2}$

Mouth cancer cases in the UK have rocketed by $39 \%$ in the last decade, and by $92 \%$ since the 1970s. Lead author, and British Association of Oral and Maxillofacial Surgeons (BAOMS) Fellow in training, David Grimes, argues that the NICE guidance (NG12) may result in delayed cancer diagnosis.

Co-author Jaymit Patel said: 'When we saw the most recent guidelines we were concerned about the recommendation for GPs to refer some patients ${ }^{\star}$ directly to dentists when no agreed pathway exists. We considered that this could lead to a delay in the review of the patient's case by an appropriately trained professional, so we decided to audit our caseload to see what the effect might be.'

David Grimes already had concerns about delays from an earlier audit he carried out where he found that half of the mouth cancer cases were diagnosed at an advanced stage. His fears were further reinforced by the report of $25 \%$ of oral cancers diagnosed at an advanced stage in the DAHNO Tenth Annual Report National Head and Neck Cancer Audit published in 2015.

Mike Bater, BAOMS Deputy oncology sub specialty interest group (SSIG) lead, said that BAOMS had raised concerns with NICE regarding the changes in the referral pathway for patients with suspected oral cancer some months ago.

The analysis of findings was published just as the Oral Health Foundation embarked on the month-long mouth cancer awareness campaign throughout November 2016. Oral Health Foundation CEO Dr Nigel Carter is urging everybody to be more Mouthaware whether they are clinicians or patients and said: 'Early diagnosis transforms a person's chances of beating the disease from $50 \%$ to $90 \%$ '.

${ }^{*}$ Some patients in this context refers to patients that meet certain criteria will be referred to a dentist, but those that do not are referred directly to hospital via the two-week wait pathway. The NICE guidance NG12 does not explicitly define the pathway or have quality control measures.

1 NICE guidance NG12. Suspected cancer: recognition and referral. June 2015. Available at https://www.nice.org.uk/guidance/ng12?unlid=9874814692015129115552,1066711945201610841519 (accessed November 2016). 2 Grimes D, Patel J, Avery C. New NICE referral guidance for oral cancer: does it risk delay in diagnosis? Br J Oral Maxillofac Surg 2016; pii: S0266-4356(16)30293-5. DOI: 10.1016/j. bjoms.2016.09.022. [Epub ahead of print].

\footnotetext{
Vacancy: Associate Editor in Science

The British Dental Association (BDA) is looking to recruit an Associate Editor in Science. This is a part-time position for one day a week, based in the BDA's offices in Wimpole Street, although candidates wishing to work more remotely can be considered. Applications must be made by 12 noon on Tuesday 2 January 2017. For the full details please visit https://www.bdjjobs. com/job/245779/associate-editor-for-science/.
}

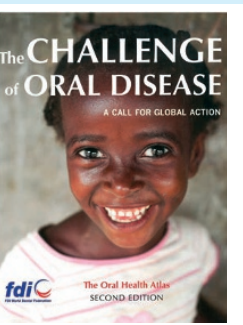

\section{THE CHALLENGE OF ORAL DISEASE: A CALL FOR GLOBAL ACTION}

FDI World Dental Federation; 2015

Price $\mathrm{f20}$; pp 120

ISBN 9782970093480

Untreated tooth decay is the most widespread chronic disease, affecting almost half of the world's population (44\%). Standardised global data on the topic is limited due to the lack of epidemiological information which tremendously hinders resources that can help to reduce the burden of disease. The Oral Health Atlas was issued in 2009. This is the follow-up edition released almost six years after the original. The Challenge of Oral Disease released by the FDI highlights promising opportunities which can help to tackle the burden of oral health issues. The content itself is well written, offering depth and background to oral health professionals and policy makers, as well as the general public about the affliction of several oral conditions. Consequently, themes on oral cancer are made more relatable through patient testimonies by public figures such as Rod Stewart and Michael Douglas. This updated version includes various additions, such as the climate of oral health, and highlights the fact that there are low allocations of resources globally for prevention, treatment and further research. The book wonderfully encompasses the current challenges faced by oral health professionals and the possible actions that can be taken to alleviate disease. It is supplemented with an overview of the history of oral health and important information is highlighted through clear tables and graphs, illustrating where research has been performed well and where it is lacking. This book is easily understandable and a real eye opener.

\section{BDA Branch AGMs}

\section{North of Scotland}

North of Scotland Branch AGM will be held at

18.45 on Wednesday 14 December at Dundee

Educational Centre, Dundee, prior to the Dundee/Perth Section meeting. All members are cordially invited to attend. Nominations are being sought for Branch President and Branch Treasurer. For further information, to confirm attendance and/or submit apologies contact Ardalan Eghtedar on ardalan.eghtedar@gmail.com.

\section{South Mercia}

South Mercia Branch AGM will be held on 25 January 2017 at the Holiday Inn, Peartree Island, Oxford. The AGM will be held prior to the Oxford section educational meeting. All members are welcome to stay for the buffet and educational meeting, but need to book onto this part of the event via Eventbrite. For further information, to confirm attendance or submit your apologies please contact Alison Lockyer at dr.arlockyer@gmail.com. 